\section{Assessment of Lower Limb Microcirculation: Exploring the Reproducibility and Clinical Application of Laser Doppler Techniques}

\author{
M. Klonizakis G. Manning R. Donnelly
}

Division of Vascular Medicine, School of Graduate Entry Medicine and Health, University of Nottingham, Nottingham, UK

\begin{tabular}{l} 
(C) Free Author \\
Copy - for per- \\
sOnal use Only \\
ANY DISTRIBUTION OF THIS \\
ARTICLE WITHOUT WRITTEN \\
CONSENT FROM S. KARGER \\
AG, BASEL ISA VIOLATION \\
OF THE COPYRIGHT. \\
Written permission to distrib- \\
ute the PDF will be granted \\
against payment of a per- \\
mission fee, which is based \\
on the number of accesses \\
required. Please contact \\
permission @ karger.ch \\
\hline
\end{tabular}

\section{Key Words}

Reproducibility • Iontophoresis • Laser Doppler fluximetry • Endothelial function - Microvascular perfusion $\cdot$ Clinical application

\begin{abstract}
Purpose of Study: Non-invasive laser Doppler fluximetry (LDF) and laser Doppler imaging (LDI), combined with iontophoresis, have been used to study the microcirculation in a range of clinical conditions including lower limb venous disease. A prerequisite for an accurate measurement tool is that it is reproducible. However, there is currently no literature with respect to the reproducibility of LDF and LDI combined with iontophoresis in the lower limb (in general) and in the upright position (in specific). Furthermore, the two techniques have been used interchangeably by researchers and the association between these two different measurement methods has not been explored, nor have the factors that affect them been well described. Thus the aim of this study was to determine the reproducibility of LDF and LDI with iontophoresis in the lower limb and investigate factors that influence their clinical application. Procedures: Cutaneous microvascular responses in the lower limb were measured in the supine and standing positions using LDF and LDI combined with iontophoretic administration of endothelial-dependent (acetylcholine, ACh) and -independent (sodium
\end{abstract}

nitroprusside) vasodilators in 25 patients with uncomplicated isolated superficial venous incompetence (ISVI) and 26 healthy controls. Results: Maximum perfusion had the best reproducibility assessed by LDF (CV 20.5-24.3\%) and LDI (15.8-17.6\%). Both techniques were positively influenced by iontophoretic dose (e.g. $p=0.0001$ for LDF) and the use of vasodilator agents (e.g. $p=0.0001$ for LDF), but negatively influenced in the standing position and/or in the presence of ISVI ( $p=0.0016$ and 0.045 , respectively, for LDF). There was a statistically significant positive relationship between the two techniques, for example ACh maximum perfusion versus LDF ACh maximum perfusion ( $r=0.404, p=0.016)$. Conclusions: Both techniques are reproducible, in line with similar studies undertaken in other areas of the human body, and provide useful information for the study of the lowerlimb microcirculation. Direct comparison between techniques based on absolute numbers should be avoided and the technique choice should be based on individual study needs.

Copyright $\odot 2011$ S. Karger AG, Basel

\section{Introduction}

Adverse changes in the microvascular circulation are associated with many chronic diseases including venous disease [1,2] and diabetes [3], leading to the development

\section{KARGER}

Fax +41613061234 E-Mail karger@karger.ch www.karger.com

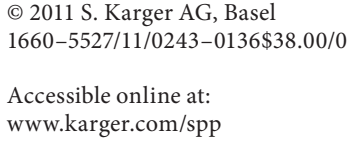

Dr. Gillian Manning, MPhil, MMedSci, PhD

School of Graduate Entry Medicine and Health

Royal Derby Hospital, Uttoxeter Road, Derby DE22 3DT (UK)

Tel. +44 1332724 682, Fax +441332 724169

E-Mail gillian.manning@ nottingham.ac.uk 
and application of a range of techniques to assess the microcirculation. For example, microangiopathic changes (in the venous microcirculation) are closely associated with trophic skin damage, and in turn such skin damage can lead to venous ulceration [4]. Non-invasive assessment of the microcirculation by laser Doppler fluximetry (LDF) and laser Doppler imaging (LDI) has been utilised as clinical surrogate marker (i.e. for systemic sclerosis [5]), to monitor postoperative tissue perfusion [6] and evaluate the effect of drugs in microcirculation [7]. Additionally, these techniques have been widely used in research in an attempt to study the effect of a range of clinical conditions in endothelial function, including, amongst others, diabetes [8], atherosclerosis [9] and lower limb venous disease [10]. Although results are promising, lack of standardised procedures and poorly defined reproducibility have limited the number of their clinical applications.

Despite the fact that these two techniques share the same principle [10] there are also differences. LDF electrodes are applied directly to the skin and typically measure within a small area $\left(\sim 1 \mathrm{~mm}^{3}\right)$ [11], potentially leading to spatial heterogeneity and artefact movement. In contrast, LDI usually measures a larger skin area from a distance without touching the tissue, generating a colourcoded image of the spatial distribution of tissue perfusion [10]. LDI is associated with a high capital and maintenance cost (currently in the order of GBP 37,500 compared to the typical GBP 8,500 cost of an LDF system) and LDI can only provide an image of perfusion at a given point. LDF, however, provides a constant measure of blood flow [12], an important requirement when studying the temporal variation of cutaneous flow.

The technique of iontophoresis is often used as an adjunct to LDF and LDI. This established, non-invasive method enables the introduction of charged substances across the surface of the skin by means of a small electric current. This allows a high concentration of drug to be delivered to a local skin site, based on the principle that an electrical potential difference will actively cause ions in solution to migrate according to their electrical charge [13]. Thus iontophoresis leads to reproducible enhancement of transdermal permeability at a level sufficient to increase the number of diagnostic and therapeutic opportunities via the skin [14].

The performance and reproducibility of these methods has been reported in a number of studies [10, 13-16], but technological improvements (e.g. in the probe design and laser light emission) have been made in recent years. Early LDF studies highlighted that the method was reli- able for the short-term investigation of drug effects on the microcirculation and for quantifying changes in the cutaneous microvascular perfusion $[15,16]$. This conclusion was drawn, despite the reported differences between proximal and distal sites of recordings [16] and intra- and interindividual differences reported to be $34 \pm 4$ and 41 $\pm 5 \%$ for acetylcholine (ACh), and $34 \pm 4$ and $29 \pm 3 \%$ for sodium nitroprusside (SNP), respectively [13]. However, the introduction of newer, more sophisticated probes (e.g. use of a single laser Doppler fibre surrounded by several receiving fibres) has improved results, as the study of a larger volume is enabled [12]. In addition, and although the reproducibility of LDF has been evaluated in other contexts (e.g. choroidal blood perfusion [17]), studies in the lower limbs have been limited [18], with no reproducibility studies, to our knowledge, been undertaken in the upright position.

Studies using LDI have reported better levels of reproducibility. For example Kubli et al. [19], in a study undertaken in the forearm, reported that the mean coefficient of variation $(\mathrm{CV})$ of responses was $<10 \%$ for $\mathrm{ACh}$ and between 10 and 20\% for SNP. Morris and Shore [20] reported slightly higher values for ACh (e.g. $23.2 \pm 12.4 \%$ ), a finding that could potentially be attributed to possible different protocol and study conditions (i.e. the study by Kubli et al. [19] included only male participants, with total current charges of 22 and $38 \mathrm{mC}$ for ACh and SNP, respectively, while Morris and Shore [20] used both male and female participants and applied 7 pulses of $0.1 \mathrm{~mA}$ for $20 \mathrm{~s}$ followed by one pulse of $0.2 \mathrm{~mA}$ for $20 \mathrm{~s}$ ).

Although the reported reproducibility of LDF and LDI has been variable, it is clear that differences in study populations, laser Doppler and iontophoresis equipment (i.e. using equipment with different penetration depth), the sites studied (i.e. upper or lower side of forearm [21]), and the conditions under which reproducibility was tested (i.e. different temperature levels) make meaningful comparisons between studies more difficult [19]. Furthermore, and although the two techniques have been used interchangeably, the association between the measurement output is unknown with the factors that affect them being poorly defined and understood.

Thus the aim of this study was to determine the reproducibility of LDF and LDI with iontophoresis in the lower limb in the supine and standing position. Additionally, we aimed to investigate factors that may influence each technique and make recommendations for future use of these techniques. 


\section{Methods}

Patients with uncomplicated isolated superficial venous incompetence (ISVI), i.e. long or short saphenous vein reflux (CEAP classification: C2-C3) confirmed by duplex scanning (conducted by a trained and accredited NHS clinical physiologist [22]), who were awaiting surgery were recruited from referrals to the Derby Royal Infirmary Vascular Clinic. Patients with present or past venous ulceration, arterial disease or major skin changes in the gaiter area were excluded. Healthy controls were recruited from the research database in the Division of Vascular Medicine, and the absence of ISVI confirmed by duplex scanning, conducted by a trained and accredited NHS Clinical Physiologist in the Derby Royal Infirmary Vascular Clinic. This research was carried out in accordance with the Declaration of Helsinki of the World Medical Association and all participants gave written informed consent. The protocol was approved by the Southern Derbyshire Local Research Ethics Committee. Sample size calculations were based on our previous work on similar study groups [23].

Following baseline screening, including measurement of height (metres), weight (kilograms), high-density lipoprotein, total cholesterol and blood pressure ( $\mathrm{mm} \mathrm{Hg}$ ), participants attended the Clinical Research Unit on two separate study mornings, 2 weeks ( \pm 2 days) apart. Microvascular perfusion and vasodilator responses in the gaiter area were measured in a temperature-controlled room according to the same, standard protocol on both study days. Measurements were undertaken in the morning hours (8.30-10.30), while participants were asked to refrain from smoking and any food, tea and coffee intake prior to the experiment.

\section{Study Periods}

\section{Laser Doppler Fluximetry}

Following $30 \mathrm{~min}$ rest with the leg supported at a $30^{\circ}$ angle above the heart level, the gaiter area was cleaned with alcohol and dried. Two Perspex iontophoresis chambers were positioned over healthy skin (avoiding any area of lipodermatosclerosis or superficial veins), approximately $2-5 \mathrm{~cm}$ apart on the surface of the leg, $4-8 \mathrm{~cm}$ proximal to the medial malleolus. Then, $1 \%$ ACh (Sigma Chemicals, UK) and 1\% SNP (Nipride, Roche Pharmaceuticals Ltd.) diluted in deionised HPLC-grade water were injected into the anodal and cathodal iontophoresis chambers, respectively, with the laser Doppler probe being positioned through the centre of each chamber. The choice of drug concentration and electric current was made, in order to minimise non-specific vasodilator effects [24].

LDF measurements were made using the DRT4 (Moor Instruments, Axminster, UK), including the skin temperature, perfusion and microvascular dose-response curves for each iontophoretic challenge obtained. After achieving a stable recording of baseline perfusion, LDF responses to ACh and SNP were measured using an incremental-dose iontophoresis protocol [1]. Thereafter, following a 10-min recovery period and the stabilisation of baseline perfusion recordings in the standing position, repeat measurements of vasodilator responses were performed, with the subject upright at exactly the same site to the one used in the supine position. The protocol was repeated on visit 2 .

\section{Laser Doppler Imaging}

LDI measurements using an LDI system (Perimed, Stockholm, Sweden) and following the same procedure and protocol as the one described above were undertaken on the same days as the LDF measurements, $2 \mathrm{~h}$ after completion of the latter and following a recovery and stabilising period.

The laser Doppler imager was adjusted to scan the area covered by the two iontophoresis chambers used to deliver the two agents, having a consistent distance from the subject's leg $(21 \mathrm{~cm})$.

A battery-powered constant current controller (Perimed) was used to provide a direct current for the drug iontophoresis. Both the duration and strength of the constant current of each dose were defined manually, according to the standard protocol described above. Perfusion responses and the obtained images were stored after the end of each experiment on the computer connected to the laser Doppler imager.

\section{Data Analysis}

Microvascular perfusion measured by LDF combined with iontophoresis was determined using three parameters: mean and maximum perfusion (measured in perfusion units), and the area under the curve (AUC, unitless, fig. 1). For LDI, the three parameters measured were minimum perfusion (volts), mean perfusion (volts) and maximum perfusion (volts). All quantities were measured at baseline and after each iontophoretic charge (250, 500, 1,000 and 2,000 $\mu \mathrm{Cb}$ ). Baseline to $2,000 \mu \mathrm{Cb}$ was defined as the difference in perfusion between these two periods.

Reproducibility was determined by comparison of the results from visit 1 and 2, for all the applied charges, both agents (ACh and SNP) and both positions (supine and standing), for each method separately.

\section{Statistical Analysis}

Data obtained using commercial software systems (Moorsoft V1, Moor Instruments, UK for LDF and Perisoft, Perimed, Sweden for LDI) was transferred to a database (Excel 2000) and analysed using statistical software (SPSS version 14, SPSS, USA). Outcome measures were first tested for normal distribution using the Kolmogorov-Smirnov goodness of fit test and normalised using logarithmic transformation if necessary before further analysis.

In order to determine the reproducibility of the techniques in the supine and standing positions concordance correlation coefficient [25] and the mean within-subject CV were calculated. CV was calculated by dividing the within-subject standard deviation (SD) by the mean and expressing it as a percentage. The withinsubject SD was calculated as the square root of the residual mean square using two-way analysis of variance. In addition Bland-Altman plots were used to assess reproducibility. Ninety-five percent of the differences between the two measurements ought to lie within $1.96 \mathrm{SD}$ of the differences if good repeatability is present [26]. Looking at the spread of points ensures that there is no relation between the magnitude of the response and the difference between the two measurements.

Wilcoxon signed-rank test (for paired samples) and Bonferroni corrections were used where appropriate. Data is presented as mean (SD). Correlation and equivalency tests (in the form of Pearson's product-moment coefficient and Fisher's exact tests) were used to determine the relationship between a number of methodological (position, vasodilator agent, dose), clinical and demographic factors (presence or absence of ISVI, gender, smok- 
Fig. 1. Typical LDF trace depicting the measurements made for each time period.

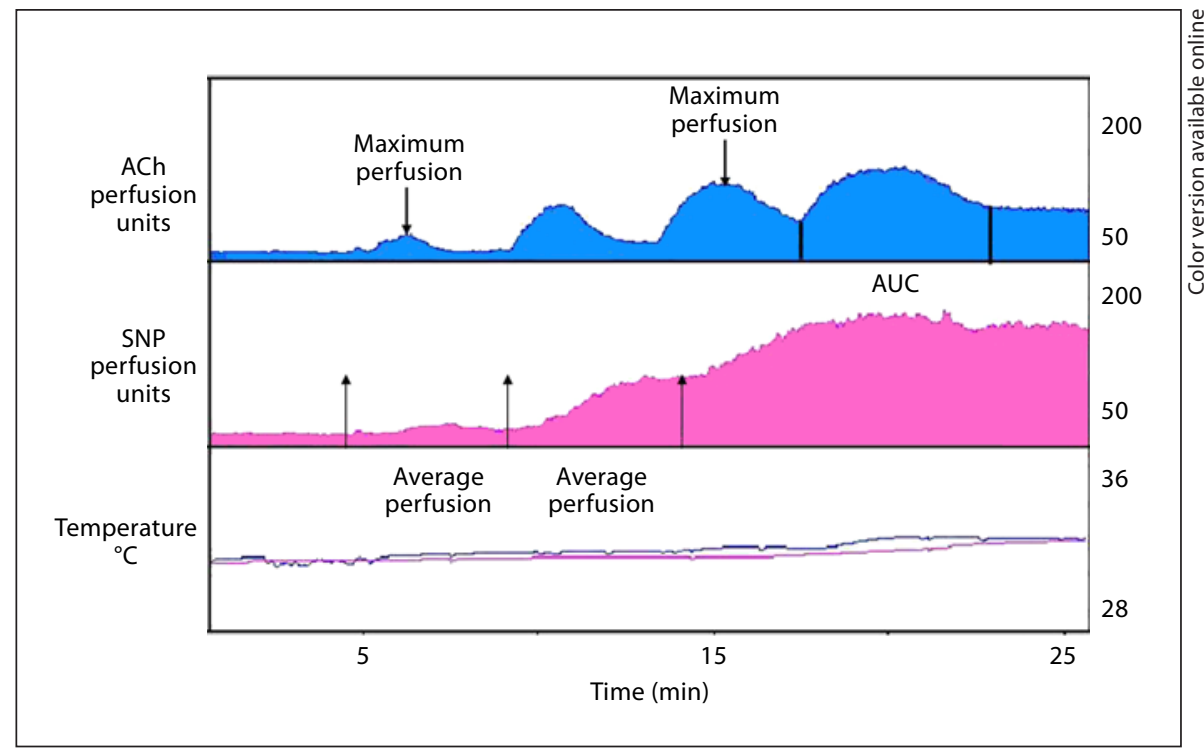

ing habits, exercise and alcohol consumption). Based on the results of these tests an analysis of covariance (ANCOVA) model was developed to determine their influence on each measure and for each laser Doppler method separately. Similarly, bivariate analysis was undertaken using Pearson's product-moment coefficient to assess associations between LDF and LDI measures of endothelial function. Correlation coefficients were characterised according to Cohen [27], e.g. small if between 0.1 and 0.3 , moderate if being between 0.3 and 0.5 and large if being $0.5-1.0$. As no well-established threshold exists, below which within-subject CV are considered as 'good' or 'excellent', we based our interpretation of the results on the work of Harris et al. [28] which suggests that CVs are acceptable if $<35 \%$.

\section{Results}

LDF and LDI were undertaken in 25 ISVI and 26 control subjects. A detailed demographic summary is presented in table 1 .

\section{Reproducibility}

$L D F$. The findings from LDF for the three measures were compared for each iontophoretic dose, agent and position between the two visits. No statistically significant differences were observed in measurements between the two visits ( $\mathrm{p}=0.77$, Wilcoxon signed-rank test). Maximum perfusion was found to be the most reproducible quantity at baseline to maximal charge (table 2, fig. 2).

LDI. Maximum and mean perfusion were found to have similar CV for LDI (ranging from 15.8 to $17.6 \%$ and
Table 1. Demographic characteristics of participants presented per group (data presented as mean $\pm \mathrm{SD}$ )

\begin{tabular}{lccc}
\hline & $\begin{array}{c}\text { Control } \\
(\mathrm{n}=26)\end{array}$ & $\begin{array}{l}\text { ISVI } \\
(\mathrm{n}=25)\end{array}$ & $\mathrm{p}$ value \\
\hline Age, years & $54 \pm 12$ & $53 \pm 11$ & 0.61 \\
BMI & $25.4 \pm 3.3$ & $25.9 \pm 3.9$ & 0.70 \\
Height, m & $1.70 \pm 0.1$ & $1.72 \pm 0.2$ & 0.71 \\
Weight, kg & $75 \pm 11$ & $78 \pm 13$ & 0.55 \\
Systolic BP, mm Hg & $125 \pm 19$ & $130 \pm 24$ & 0.32 \\
Diastolic BP, mm Hg & $78 \pm 8$ & $81 \pm 9$ & 0.34 \\
Total cholesterol, mmol/1 & $5.2 \pm 1.4$ & $5.6 \pm 1.3$ & 0.30 \\
High density lipoprotein, & & & \\
$\quad$ mmol/l & $1.52 \pm 0.4$ & $1.59 \pm 0.5$ & 0.29 \\
\hline
\end{tabular}

$\mathrm{BMI}=$ Body mass index $; \mathrm{BP}=$ blood pressure

from 15.2 to $18.7 \%$, respectively), with minimum perfusion having the lowest reproducibility (table 2). No statistically significant differences were observed in measurements between the two visits, for both agents and positions studied ( $p=0.80$, Wilcoxon signed-rank test). A sample Bland-Altman plot is presented in figure 3.

\section{Perfusion and AUC Determinants}

Separate ANCOVA models were developed to determine the factors that affect the different LDF and LDI measurement quantities. As our previous studies have in- 


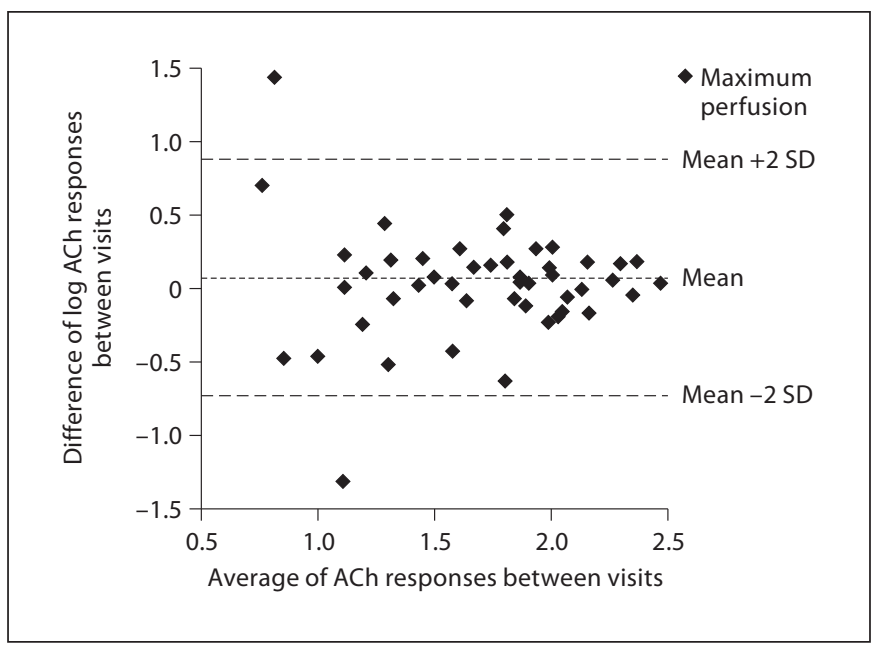

Fig. 2. Bland-Altman plot of maximum perfusion for 'baseline to maximal charge' (LDF).

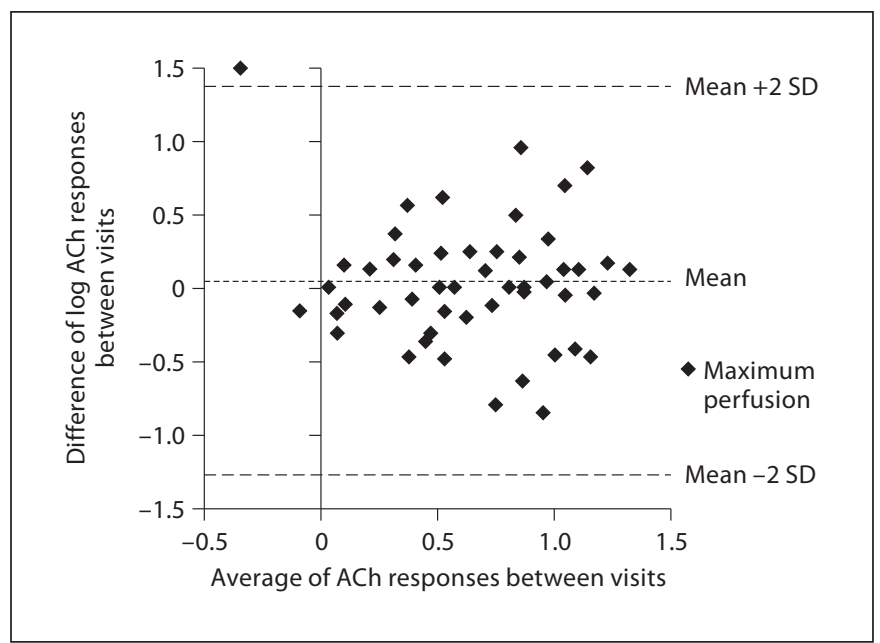

Fig. 3. Bland-Altman plot of maximum perfusion for 'baseline to maximal charge' (LDI).

Table 2. The reproducibility of LDF and LDI measurements

\begin{tabular}{|c|c|c|c|c|c|c|c|c|}
\hline \multirow[t]{2}{*}{ Quantity } & \multicolumn{2}{|c|}{ ACh lying } & \multicolumn{2}{|c|}{ ACh standing } & \multicolumn{2}{|c|}{ SNP lying } & \multicolumn{2}{|c|}{ SNP standing } \\
\hline & $\mathrm{CV}, \%$ & CCC & $\mathrm{CV}, \%$ & CCC & $\mathrm{CV}, \%$ & CCC & $\mathrm{CV}, \%$ & CCC \\
\hline AUC (LDF) & 28.4 & 0.68 & 29.6 & 0.64 & 30.7 & 0.64 & 31.8 & 0.58 \\
\hline Mean perfusion (LDF) & 24.2 & 0.76 & 25.2 & 0.68 & 21.4 & 0.72 & 22.9 & 0.70 \\
\hline Maximum perfusion (LDF) & 21.1 & 0.79 & 21.6 & 0.78 & 20.5 & 0.74 & 24.3 & 0.72 \\
\hline Minimum perfusion (LDI) & 27.5 & 0.59 & 26.7 & 0.59 & 29.4 & 0.54 & 28.7 & 0.55 \\
\hline Mean perfusion (LDI) & 18.7 & 0.71 & 17.7 & 0.78 & 15.2 & 0.84 & 16.4 & 0.80 \\
\hline Maximum perfusion (LDI) & 17.6 & 0.81 & 15.8 & 0.83 & 15.9 & 0.82 & 17.4 & 0.80 \\
\hline
\end{tabular}

$\mathrm{CCC}=$ Concordance correlation coefficient.

ferred $[1,9,23]$, LDF measurement quantities are positively influenced by iontophoretic dose and the use of vasodilator agents and reduced in the standing position and when ISVI is present (table 3). Similar results were obtained during LDI measurements, with all three quantities being simultaneously influenced by the same factors. Conversely, gender was not found to be of significance for any measurement for both techniques, while smoking did not have a statistically significant effect on maximum perfusion, despite the fact that it affects mean perfusion and AUC in LDF and mean and minimum perfusion in LDI.

\section{Associations between Measurements and Techniques}

The exploration of the associations between LDF and LDI measures was divided in two sections (table 4).
With respect to the intra-technique relationship, statistically significant correlations were identified between LDI measures in ACh vasodilation measurements: e.g. LDI maximum perfusion with LDI mean perfusion $(\mathrm{r}=$ 0.763, $\mathrm{p}<0.001)$. Similarly for LDF, Maximum Perfusion was strongly correlated with AUC $(\mathrm{r}=0.78, \mathrm{p}<$ $0.001)$ and Mean Perfusion with AUC ( $r=0.925, p<$ $0.001)$.

Intertechnique associations between different measures of microvascular vasodilator function were significant, yet generally, moderate [27] at best: i.e. LDI maximum perfusion with LDF maximum perfusion $(\mathrm{r}=0.404$, $\mathrm{p}=0.016)$. 
Table 3. Relationship between LDF and LDI measures and determinants

\begin{tabular}{|c|c|c|c|c|c|c|}
\hline \multirow[t]{2}{*}{ Quantity } & \multicolumn{3}{|l|}{$\mathrm{LDF}$} & \multicolumn{3}{|l|}{ LDI } \\
\hline & maximum & mean & AUC & maximum & mean & minimum \\
\hline \multicolumn{7}{|l|}{ Determinant } \\
\hline Iontophoretic dose & 0.0001 & 0.0001 & 0.0001 & 0.0001 & 0.0001 & 0.001 \\
\hline Position & 0.0001 & 0.0001 & 0.0001 & 0.0001 & 0.0001 & 0.0001 \\
\hline Vasodilatory agents & 0.045 & 0.001 & 0.043 & 0.03 & 0.04 & 0.045 \\
\hline Presence of ISVI & 0.016 & 0.039 & 0.022 & 0.02 & 0.03 & 0.02 \\
\hline Gender & NS & NS & NS & NS & NS & NS \\
\hline Alcohol & NS & NS & NS & NS & NS & NS \\
\hline Training & NS & NS & NS & NS & NS & NS \\
\hline Smoking & NS & 0.012 & 0.037 & NS & 0.015 & 0.041 \\
\hline
\end{tabular}

Table 4. Associations between LDF and LDI measures (ACh and SNP)

\begin{tabular}{|c|c|c|c|c|c|c|c|}
\hline & & $\begin{array}{l}\text { Mean } \\
\text { perfusion } \\
(\mathrm{LDI})\end{array}$ & $\begin{array}{l}\text { Maximum } \\
\text { perfusion } \\
\text { (LDI) }\end{array}$ & $\begin{array}{l}\text { Minimum } \\
\text { perfusion } \\
\text { (LDI) }\end{array}$ & $\begin{array}{l}\text { Maximum } \\
\text { perfusion } \\
(\mathrm{LDF})\end{array}$ & $\begin{array}{l}\text { AUC } \\
(\mathrm{LDF})\end{array}$ & $\begin{array}{l}\text { Mean } \\
\text { perfusion } \\
(\mathrm{LDF})\end{array}$ \\
\hline \multicolumn{8}{|l|}{$A C h$} \\
\hline \multirow{2}{*}{$\begin{array}{l}\text { Mean perfusion } \\
(\mathrm{LDI})\end{array}$} & \multirow{2}{*}{$\begin{array}{l}\text { Pearson correlation } \\
\text { sig. ( } 2 \text {-tailed) }\end{array}$} & - & $0.763^{* *}$ & $0.517^{* *}$ & -0.056 & -0.016 & 0.085 \\
\hline & & - & 0.000 & 0.001 & 0.746 & 0.924 & 0.616 \\
\hline \multirow{2}{*}{$\begin{array}{l}\text { Maximum perfusion } \\
\text { (LDI) }\end{array}$} & \multirow{2}{*}{$\begin{array}{l}\text { Pearson correlation } \\
\text { sig. ( } 2 \text {-tailed) }\end{array}$} & $0.763^{* *}$ & - & $0.394^{*}$ & $0.404^{*}$ & $0.414^{*}$ & $0.345^{*}$ \\
\hline & & 0.000 & - & 0.048 & 0.016 & 0.013 & 0.042 \\
\hline \multirow{2}{*}{$\begin{array}{l}\text { Minimum perfusion } \\
\text { (LDI) }\end{array}$} & \multirow{2}{*}{$\begin{array}{l}\text { Pearson correlation } \\
\text { sig. (2-tailed) }\end{array}$} & $0.517^{* *}$ & $0.394^{*}$ & - & 0.045 & 0.223 & $0.449^{* *}$ \\
\hline & & 0.001 & 0.048 & - & 0.793 & 0.197 & 0.007 \\
\hline \multirow{2}{*}{$\begin{array}{l}\text { Maximum perfusion } \\
\text { (LDF) }\end{array}$} & \multirow{2}{*}{$\begin{array}{l}\text { Pearson correlation } \\
\text { sig. }(2 \text {-tailed) }\end{array}$} & -0.056 & $0.404^{*}$ & 0.045 & - & $0.781^{* *}$ & -0.265 \\
\hline & & 0.746 & 0.016 & 0.793 & - & 0.000 & 0.124 \\
\hline \multirow{2}{*}{$\begin{array}{l}\text { AUC } \\
\text { (LDF) }\end{array}$} & \multirow{2}{*}{$\begin{array}{l}\text { Pearson correlation } \\
\text { sig. (2-tailed) }\end{array}$} & 0.328 & $0.414^{*}$ & 0.223 & $0.781^{* *}$ & - & $0.925^{* *}$ \\
\hline & & 0.054 & 0.013 & 0.197 & 0.000 & - & 0.000 \\
\hline \multirow{2}{*}{$\begin{array}{l}\text { Mean perfusion } \\
(\mathrm{LDF})\end{array}$} & \multirow{2}{*}{$\begin{array}{l}\text { Pearson correlation } \\
\text { sig. ( } 2 \text {-tailed) }\end{array}$} & $0.471^{* *}$ & $0.345^{*}$ & $0.449^{* *}$ & -0.265 & $0.925^{* *}$ & - \\
\hline & & 0.004 & 0.042 & 0.007 & 0.124 & 0.000 & - \\
\hline \multirow{3}{*}{$\begin{array}{l}S N P \\
\text { Mean perfusion } \\
\text { (LDI) }\end{array}$} & \multirow{3}{*}{$\begin{array}{l}\text { Pearson correlation } \\
\text { sig. ( } 2 \text {-tailed) }\end{array}$} & & & & & & \\
\hline & & - & $0.654^{* *}$ & 0.200 & 0.109 & 0.147 & -0.169 \\
\hline & & - & 0.000 & 0.248 & 0.547 & 0.413 & 0.338 \\
\hline \multirow{2}{*}{$\begin{array}{l}\text { Maximum perfusion } \\
\text { (LDI) }\end{array}$} & \multirow{2}{*}{$\begin{array}{l}\text { Pearson correlation } \\
\text { sig. }(2 \text {-tailed })\end{array}$} & $0.654^{* *}$ & - & 0.133 & -0.169 & 0.109 & 0.147 \\
\hline & & 0.000 & - & 0.432 & 0.338 & 0.547 & 0.413 \\
\hline \multirow{2}{*}{$\begin{array}{l}\text { Minimum perfusion } \\
\text { (LDI) }\end{array}$} & \multirow{2}{*}{$\begin{array}{l}\text { Pearson correlation } \\
\text { sig. }(2 \text {-tailed })\end{array}$} & 0.200 & 0.133 & - & 0.192 & 0.188 & -0.006 \\
\hline & & 0.248 & 0.432 & - & 0.270 & 0.280 & 0.971 \\
\hline \multirow{2}{*}{$\begin{array}{l}\text { Maximum perfusion } \\
\text { (LDF) }\end{array}$} & \multirow{2}{*}{$\begin{array}{l}\text { Pearson correlation } \\
\text { sig. (2-tailed) }\end{array}$} & 0.109 & -0.169 & 0.192 & - & $0.924^{* *}$ & 0.235 \\
\hline & & 0.547 & 0.338 & 0.270 & - & 0.000 & 0.140 \\
\hline \multirow{2}{*}{$\begin{array}{l}\text { AUC } \\
(\mathrm{LDF})\end{array}$} & \multirow{2}{*}{$\begin{array}{l}\text { Pearson correlation } \\
\text { sig. }(2 \text {-tailed })\end{array}$} & 0.147 & 0.109 & 0.188 & $0.924^{* *}$ & - & 0.297 \\
\hline & & 0.413 & 0.547 & 0.280 & 0.000 & - & 0.098 \\
\hline \multirow{2}{*}{$\begin{array}{l}\text { Mean perfusion } \\
\text { (LDF) }\end{array}$} & \multirow{2}{*}{$\begin{array}{l}\text { Pearson correlation } \\
\text { sig. ( } 2 \text {-tailed) }\end{array}$} & -0.169 & 0.147 & -0.006 & 0.235 & 0.297 & - \\
\hline & & 0.338 & 0.413 & 0.971 & 0.140 & 0.098 & - \\
\hline
\end{tabular}

** Correlation is significant at the 0.01 level (2-tailed).

* Correlation is significant at the 0.05 level (2-tailed). 


\section{Discussion}

Assessment of microvascular function is clinically important across a range of conditions including venous disease. Recent refinements in LDF equipment including the calculation algorithms [29] have led to researchers $[16,18,30]$ supporting the use of LDF as an effective and useful tool for evaluating the cutaneous microcirculation, provided that standardised procedures are followed $[12,29]$. Given that an assessment tool should be reproducible, but the microvascular beds differ within the human circulation, this study provides important new information with respect to the reproducibility of LDF and LDI in the lower limb [19].

Analysis of the three commonly used LDF parameters measuring microvascular perfusion and reactivity showed that maximum perfusion had the highest reproducibility, with AUC having the lowest. With respect to LDI, maximum perfusion and mean perfusion were the most reproducible measures, whilst the minimum perfusion was found to be the least reproducible.

Direct comparison between LDI reproducibility studies can be very difficult [10] but our findings are in general agreement with previous reproducibility studies undertaken in the upper limbs in normal subjects $[10,19]$ and in patient populations [21]. This suggests that the combined use of LDI and iontophoresis is reproducible, provided the recording sites are standardised and adherence to a protocol is maintained.

This is the first study to examine the reproducibility of both techniques and their standard measurement quantities in the upright position. Our findings suggest that using our standardised protocol the reproducibility in the standing position is similar to that obtained in the supine position (table 2); we hope this study will pave the way for more studies in the upright position.

In general, assessment by LDI was more reproducible than LDF. It is possible that the higher CV observed in LDF is affected by the dermal perfusion variations that occur within $\sim 1 \mathrm{~mm}^{2}$ of skin, which is the area that the LDF probe operates on [11], or the choice of measurement site (gaiter area), as this is one of the factors affecting LDF measurements [12]. This could also reflect the fact that LDI involves simultaneous perfusion measurements in a very large number of points. The latter suggests that a direct comparison between the two techniques based on absolute numbers should be avoided. We would recommend that LDF should be used for studies requiring continuous measurements with LDI being utilised as a means of studying larger surface areas. The lack of strong asso- ciation between the two techniques further supports this recommendation and suggests that the techniques cannot be used interchangeably, an argument supported in the literature [31].

The final part of this study explored the effects of a range of clinical parameters on the LDF and LDI measurements. Iontophoretic dose, bodily position, vasodilator agents, the presence of ISVI and smoking were more likely to influence both LDF and LDI measurements. Further work is needed to clarify the importance of this finding, however, it can be postulated that the combination of smoking and ISVI can be a very serious inhibitor of microvascular perfusion and reactivity.

This study is the first to our knowledge to directly compare LDF and LDI combined with iontophoresis in both supine and standing positions in the lower limb and assess the reproducibility of standard measures, assessing their inter- and intratechnique associations and exploring factors that affect them. We have shown that both LDF and LDI, in both positions, have a reproducibility similar to if not better than other biological measurements $[32,33]$ and, therefore, can be used in the study of the pathophysiology in a number of conditions affecting microcirculation (e.g. ISVI, diabetes) without any debate about their applicability. As we have successfully presented on this occasion by studying the effect of a number of methodological, clinical and demographic factors, perfusion measurements are affected by a combination of conditions and parameters (e.g. ISVI, smoking and prolonged standing), an observation that can be helpful in the provision of clinical recommendations.

References

1 Klonizakis M, Yeung JM, Lingam K, Nash JR, Manning G, Donnelly R: Contrasting effects of varicose vein surgery on endothelialdependent and -independent cutaneous vasodilation in the perimalleolar region. Eur J Vasc Endovasc Surg 2006;31:434-438.

2 Rossi M, Carpi A, Galetta F, Franzoni F, Santoro G: Skin vasomotion investigation: a useful tool for clinical evaluation of microvascular endothelial function? Biomed Pharmacother 2008;62:541-545.

3 Khan F, Elhadd TA, Greene SA, Belch JJF: Impaired skin microvascular function in children, adolescents and young adults with type 1 diabetes. Diabetes Care 2000;23:215220

4 Robertson L, Lee AJ, Gallagher K, Carmichael SJ, Evans CJ, McKinstry BH, Fraser SC, Allan PL, Weller D, Ruckley CV, Fowkes FG: Risk factors for chronic ulceration in patients with varicose veins: a case control study. J Vasc Surg 2009;49:1490-1498. 
5 Wigley FM, Wise RA, Mikdashi J, Schaefer $\mathrm{S}$, Spence RJ: The post-occlusive hyperemic response in patients with systemic sclerosis. Arthritis Rheum 1990;33:1620-1625.

6 Place MJ, Witt P, Hendricks D: Cutaneous blood-flow patterns in free flaps determined by laser Doppler flowmetry. J Reconstr Microsurg 1996;12:355-358.

7 Binggeli C, Spieker LE, Corti R, Sudano I, Stojanovic V, Hayoz D, Lüscher TF, Noll G: Statins enhance postischemic hyperemia in the skin circulation of hypercholesterolemic patients: a monitoring test of endothelial dysfunction for clinical practice? J Am Coll Cardiol 2003;42:71-77.

8 Bonetti PO, Lerman LO, Lerman A: Endothelial dysfunction: a marker of atherosclerotic risk. Arterioscler Thromb Vasc Biol 2003;23:168-175

9 Klonizakis M, Tew G, Michaels J, Saxton J: Impaired microvascular endothelial function is restored by acute lower-limb exercise in post-surgical varicose vein patients. Microvasc Res 2009;77:158-162.

10 Svedman C, Cherry GW, Strigini E, Ryan TJ: Laser Doppler imaging of skin microcirculation. Acta Derm Venereol (Stockh) 1998;78: 114-118.

11 Braverman IM, Keh A, Goldminz D: Correlation of laser Doppler wave patterns with underlying microvascular anatomy. J Invest Dermatol 1990;95:283-286.

12 Cracowski JL, Minson CT, Salvat-Melis M, Halliwill JR: Methodological issues in the assessment of skin microvascular endothelial function in humans. Trends Pharmacol Sci 2006;27:503-508.

13 Noon JP, Walker BR, Hand MF, Webb DJ: Studies with iontophoretic administration of drugs to human dermal vessels in vivo: cholinergic vasodilatation is mediated by dilator prostanoids rather than nitric oxide. $\mathrm{Br} \mathrm{J}$ Clin Pharmacol 1998;45:545-550.
14 Guy RG: Iontophoresis-recent developments. Int J Pharm 1998;50:371-374.

15 Muller P, Keller R, Imhof P: Laser Doppler flowmetry, a reliable technique for measuring pharmacologically induced changes in cutaneous blood flow? Methods Find Exp Clin Pharmacol 1987;9:409-420.

16 De Boer EM, Bezemer PD, Bruynzeel DP: A standard method for repeated recording of skin blood flow using Laser Doppler flowmetry. Derm Beruf Umwelt 1989;37:58-62.

17 Gugleta K, Orgül S, Flammer I, Gherghel D, Flammer J: Reliability of confocal choroidal laser Doppler flowmetry. Invest Ophthalmol Vis Sci 2002;43:723-728.

18 Kvernebo K, Slagsvold CE, Stranden E, Kroese A: Laser Doppler flowmetry in evaluation of lower limb resting skin circulation: a study in healthy controls and atherosclerotic patients. Scand J Clin Lab Invest 1988;48: 621-626.

19 Kubli S, Waeber B, Dalle-Ave A, Feihl F: Reproducibility of laser Doppler imaging of skin blood flow as a tool to assess endothelial function. J Cardiovasc Pharmacol 2000; 36:640-648

20 Morris SJ, Shore AC: Skin blood flow responses to the iontophoresis of acetylcholine and sodium nitroprusside in man: possible mechanisms. J Physiol 1996;496:531-542.

21 Brocx KA, Drummond PD: Reproducibility of cutaneous microvascular function assessment using laser Doppler flowmetry and acetylcholine iontophoresis. Skin Pharmacol Physiol 2009;22:313-321.

22 Talbot SR: B-mode evaluation of peripheral arteries and veins; in Zwiebel WJ: Introduction to Vascular Ultrasonography. New York, Grune \& Stratton, 1986, p 351.

23 Klonizakis M, Yeung JM, Lingam K, Nash JR, Manning G, Donnelly R: Effects of posture and venous insufficiency on endothelial-dependent and -independent cutaneous vasodilation in the perimalleolar region. Eur J Vasc Endovasc Surg 2003;26:100-104.
24 Droog EJ, Sjöberg F: Nonspecific vasodilatation during transdermal iontophoresis: the effect of voltage over the skin. Microvasc Res 2003:65:172-178.

25 Li KIL: A concordance correlation coefficient to evaluate reproducibility. Biometrics 1989;45:255-268.

26 Bland JM, Altman DG: Statistical methods for assessing agreement between two methods of clinical measurement. Lancet 1986; i:307-310.

27 Cohen J: Statistical Power Analysis for the Behavioral Sciences, ed 2. Hillsdale, Erlbaum, 1988.

28 Harris RA, Padilla J, Hanlon KP, Rink LD, Wallace JP: Reproducibility of the Flow-mediated dilation response to acute exercise in overweight men. Ultrasound Med Biol 2007; 33:1579-1585.

29 Schabauer AM, Rooke TW: Cutaneous laser Doppler flowmetry: applications and findings. Mayo Clin Proc 1994;69:564-574.

30 Andreassen AK, Gullestad L, Holm T, Simonsen S, Kvernebo K: Endothelium-dependent vasodilatation of the skin microcirculation in heart transplant recipients. Clin Transpl 1998;12:324-332.

31 Dhindsa M, Sommerlad SM, DeVan AE, Barnes JN, Sugawara J, Ley O, Tanaka H: Interrelationships among noninvasive measures of postischemic macro- and microvascular reactivity. J Appl Physiol 2008; 105: 427-432.

32 Stergiou GS, Kollias A, Rarra VC, Roussias LG: Ambulatory arterial stiffness index: reproducibility of different definitions. Am J Hypertens 2010;23:129-134.

33 de Simone G, Roman MJ, Koren MJ, Mensah GA, Ganau A, Devereux RB: Stroke volume/ pulse pressure ratio and cardiovascular risk in arterial hypertension. Hypertension 1999; 33:800-805. 